\title{
PLANETARY AND LIGHT MOTIONS FROM NEWTONIAN THEORY: AN AMUSING EXERCISE
}

\author{
K.K. Nandi ${ }^{1}$ \\ Department of Mathematics, University of North Bengal, Siliguri, WB 734430, \\ India \\ N.G. Migranov ${ }^{2}$ \\ Department of Physics, Bashkir State Pedagogical University, 3-A, October \\ Revolution Street, Ufa 450000, Bashkortostan, Russia \\ J.C. Evans ${ }^{3}$ \\ Department of Physics, University of Puget Sound, Tacoma, WA 98416, USA \\ M.K. Amedeker ${ }^{4}$ \\ Department of Physics, University of Education, Winneba, Ghana
}

\footnotetext{
${ }^{1}$ E-mail address: kamalnandi1952@yahoo.co.in

${ }^{2}$ E-mail address: migranov@bspu.ru

${ }^{3}$ E-mail address: jcevans@ups.edu

${ }^{4}$ E-mail address: mawuden@yahoo.com
}

\begin{abstract}
We attempt to see how closely we can formally obtain the planetary and light path equations of General Relativity by employing certain operations on the familiar Newtonian equation. This article is intended neither as an alternative to nor as a tool for grasping Einstein's General Relativity. Though the exercise is understandable by readers at large, it is especially recommended to the teachers of Relativity for an appreciative understanding of its peculiarity as well as its pedagogical value in the teaching of differential equations.
\end{abstract}

Everyone knows Newton's theory of gravity and some know Einstein's theory of General Relativity (GR). Undoubtedly, GR is one of the most beautiful self-consistent modern creations in the realm of theoretical physics. It has wonderfully tested against various astronomical observations to date including those in the Solar system. However, at a popular level, a naive question is often asked as to whether the GR effects could have been interpreted using a more mundane theory than the abstract theory of GR in which gravity - which is as real a force as any other - has been "geometrized". For instance, some ask the question: What is the difference between the bending of light rays in GR with that occuring in a refractive optical medium? The answer lies in the well known fact that 
the propagation of light rays in a gravity field a la GR can be exactly rephrased as propagation in an equivalent optical refractive medium with appropriate constitutive equations [1]. The refractive index can be employed in a new set of optical-mechanical equations so that a single equation covers motions of both massive and massless particles in a spherically symmetric field [2-4]. An approach of this kind provides a useful and interesting window to look at familiar observed GR results but, by no means, implies a replacement of GR.

The whole point of the above paragraph is that one inevitably needs to know the metric solutions of GR in advance. Only after knowing them, one can derive appropriate refractive indices and the method of optical-mechanical analogy in terms of these indices then exactly reproduces the GR geodesic equations. That is to say, we might employ different working methods but the physical content remains essentially that of GR. (There have been attempts to set aside GR altogether and propose alternative physics by introducing a variable test mass [5], or even assuming variable speeds of light in flat space [6]. These ideas have their own values and we are not going to discuss them here.)

The object of the present article is somewhat different: We are not going to suggest any working method of the kind described above, but present an interesting calculation. (However, it must not be weighed against the grand edifice of GR). Using PPN-like approximations on the Newtonian theory, we shall formally obtain planetary and light path equations. They resemble the path equations of GR only fortuitously and this is the amusing part. Apart from this, the contents could be instructive in exemplifying the role of numerically smaller terms in the differential equations.

To begin with, one recalls an earlier discussion of MØller [7] that has shown that the bending of light rays is due partly to the geometrical curvature of space and partly to the variation of light speed in a Newtonian potential. In fact, the ratio is exactly 50:50. The GR null trajectory equations can be integrated, once assuming a Euclidean space with a variable light speed and again a curved space with a constant light speed. This analysis and arguments clearly elucidate the complementary roles of curved space and Newtonian theory in the best possible manner. This complementarity motivates us to examine how far, if at all, we are able to introduce curvature effects in the path equations of the Newtonian theory. That is: We try to obtain, from the familiar Newtonian theory itself, the form of the known GR path equations of motion without geometrizing gravity. (It is known that the gravitational redshift is a prediction of GR, but it is also known that it can be predicted from the Equivalence Principle without using GR equations [8]. Hence we shall not address this result here.)

Let us start from the usual Kepler problem of a massive test particle moving around a spherical gravitating mass $M$ under the Newtonian inverse square law. Let $T$ and $V$ denote the kinetic and potential energies respectively. Then $T+V=$ constant $=\frac{E_{0}}{2}$ (say) implies in relativistic units

$$
\frac{1}{2}\left[\dot{r}^{2}+r^{2} \dot{\varphi}^{2}\right]-m c_{0}^{2} r^{-1}=\frac{E_{0}}{2}
$$

where $m=G M c_{0}^{-2}$ and a dot denotes differentiation with respect to Newtonian time $t, c_{0}$ is the speed of light in vacuum. The central nature of the force implies constancy of the angular momentum (the Lagrangian is independent of $\varphi$ ) such that

$$
r^{2} \dot{\varphi}=h_{0}
$$


With $u=\frac{1}{r}$, we can rewrite Eq.(1) as

$$
h_{0}^{2}\left[u^{2}+\left(\frac{d u}{d \varphi}\right)^{2}\right]-2 m u c_{0}^{2}=E_{0}
$$

where the constant $E_{0}$ has the dimension of $c_{0}^{2}$. For bound material orbits $E_{0}$ $<0$. Customarily, by differentiating again with respect to $\varphi$, one finds a second order differential equation that yields a Keplerian ellipse given by

$$
u=\frac{1}{p}(1+e \cos \varphi)
$$

where $e$ is the eccentricity, $p=\frac{h_{0}^{2}}{G M}$ is the semi-latus rectum.

Let us redefine the radial variable $u \rightarrow u^{\prime}$ through the equations

$$
\begin{gathered}
u^{\prime}=u \Phi(u) \\
\Phi(u)=\left(1+\frac{m u}{2}\right)^{-2} \\
u^{\prime}=\frac{1}{r^{\prime}} .
\end{gathered}
$$

(Aside: These transformations are not unfamiliar to those conversant with GR.) After some straightforward algebra, we get

$$
d u^{\prime}=\Phi(u) \Omega(u) d u
$$

where

$$
\begin{gathered}
\Omega(u)=\left(1+\frac{m u}{2}\right)^{-1}\left(1-\frac{m u}{2}\right) \\
\Omega\left(u^{\prime}\right)=\left(1-2 m u^{\prime}\right)^{\frac{1}{2}} \\
\Phi\left(u^{\prime}\right)=\frac{1}{4}\left[1+\left(1-2 m u^{\prime}\right)^{\frac{1}{2}}\right]^{2} .
\end{gathered}
$$

Note that $\Phi(u)$ of Eq.(6) is numerically the same as $\Phi\left(u^{\prime}\right)$ of Eq.(11). The same applies between $\Omega(u)$ of Eq.(9) and $\Omega\left(u^{\prime}\right)$ of Eq.(10). The following expansions can also be directly verified:

$$
2 m u=2 m u^{\prime}+2 m^{2} u^{\prime 2}+5 m^{3} u^{\prime 3}+\ldots=2 m u^{\prime}+O\left(m^{2} u^{\prime 2}\right) .
$$

This implies that, to first order, $r \simeq r^{\prime}$. Also,

$$
\Phi^{2}\left(u^{\prime}\right) \Omega^{2}\left(u^{\prime}\right)=1-4 m u^{\prime}+O\left(m^{2} u^{\prime 2}\right) .
$$

Let us now express Eq.(3) in terms of the new variable $u^{\prime}$. Multiplying both sides of Eq.(3) by $\Phi^{2} \Omega^{2}$ and using Eqs.(5)-(13), we get

$$
h_{0}^{2}\left[\Omega^{2} u^{\prime 2}+\left(\frac{d u^{\prime}}{d \varphi}\right)^{2}\right]=c_{0}^{2}\left[E_{0} c_{0}^{-2}+2 m u^{\prime}+O\left(m^{2} u^{\prime 2}\right)\right] \Phi^{2} \Omega^{2} .
$$


Simplifying further using Eqs.(10) and (13), we have

$$
h_{0}^{2}\left[u^{\prime 2}+\left(\frac{d u^{\prime}}{d \varphi}\right)^{2}-2 m u^{\prime 3}\right]=c_{0}^{2}\left[E_{0} c_{0}^{-2}+2 m u^{\prime}\left(1-2 E_{0} c_{0}^{-2}\right)+O\left(m^{2} u^{\prime 2}\right)\right] .
$$

Apply this equation to a practical situation, the Solar system. At the site of Mercury, the planet nearest to the Sun, $m u \simeq m u^{\prime} \simeq 2.5 \times 10^{-8}$. Let us ignore the terms $O\left(m^{2} u^{\prime 2}\right)$ in comparison to the $m u^{\prime}$ term. Then Eq.(15) reduces to

$$
h_{0}^{2}\left[u^{\prime 2}+\left(\frac{d u^{\prime}}{d \varphi}\right)^{2}-2 m u^{\prime 3}\right]=E_{0}+2 m u^{\prime} c_{0}^{2}\left(1-2 E_{0} c_{0}^{-2}\right) .
$$

Differentiating with respect to $\varphi$, we get

$$
u^{\prime}+\frac{d^{2} u^{\prime}}{d \varphi^{2}}=\frac{1}{p^{\prime}}+3 m u^{\prime 2}
$$

where

$$
\frac{1}{p^{\prime}}=\frac{m c_{0}^{2}}{h^{\prime 2}}, h^{\prime}=\frac{h_{0}}{\left(1-2 E_{0} c_{0}^{-2}\right)^{\frac{1}{2}}}
$$

is a rescaled constant.

The final Eq.(17) seems suggestive with the usual perturbation term $3 m u^{\prime 2}$ appearing: It is exactly of the same form as the GR path equation! One notes that the constant $h^{\prime}$ involves the test particle energy $E_{0}$ similar to what one finds in the GR treatment. To see this, compare with Eq.(17) the corresponding GR equation given by (Take henceforth $G=1$ ):

$$
u+\frac{d^{2} u}{d \varphi^{2}}=\frac{1}{p}+3 m u^{2}
$$

in which $p$ is given by $p=\frac{U_{3}^{2}}{M m_{0}^{2} c_{0}^{4}}$ where $m_{0}$ is the test particle rest mass, $J=-\frac{U_{3}}{U_{0}}$ is the constant angular momentum rescaled by the energy at infinity $U_{0}=\frac{m_{0} c_{0}^{2}}{\sqrt{1-\dot{r}_{\infty}^{2} / c_{0}^{2}}}$ and the constant $U_{3}=r^{2} \frac{d \varphi}{d \lambda}, \lambda$ being the affine pararneter [9]. As usual, considering low velocity, we can take $U_{0}=m_{0} c_{0}^{2}$ and identifying the asymptotic value of $J$ as $h_{0}$, we have

$$
p \simeq \frac{h_{0}^{2}}{M} .
$$

With this value of $p$, the GR perturbation term $3 m u^{2}$ then gives the well known perihelion advance of the Keplerian ellipse.

In our case, the parallel of $p$ from Eq.(17) is:

$$
p^{\prime}:=\left(\frac{m c_{0}^{2}}{h^{\prime 2}}\right)^{-1}=\frac{h_{0}^{2}}{M\left(1-2 E_{0} c_{0}^{-2}\right)} .
$$

Its asymptotic value can be computed using Eq.(1). For near circular orbits, the kinetic and potential energies are roughly of the same order of magnitude such that the velocity is $v^{2} \sim \frac{M}{r}=m u c_{0}^{2}$. Then, from Eq.(1), and noting that 
$u \simeq u^{\prime}$ asymptotically, we can write $E_{0}=\alpha m u^{\prime} c_{0}^{2}$ where $-1<\alpha<1$. Then the denominator becomes $M\left(1-2 \alpha m u^{\prime}\right)$. The term $2 \alpha m u^{\prime} \simeq 10^{-8}$ can be easiliy ignored compared to unity and we are left with

$$
p^{\prime} \simeq \frac{h_{0}^{2}}{M}
$$

just as in Eq.(20). So we can replace $p^{\prime}$ in Eq.(17) by its asymptotic value $p$ given either by Eq.(20) or (22).

For the motion of light, the situation is different: the dimensionless quantity $E_{0} c_{0}^{-2}$ must be fixed to the value $\frac{1}{2}$ so that $p^{\prime} \rightarrow \infty$. Recall that only a nonzero value for light $\left(E_{0} \neq 0\right)$ in Newtonian theory is consistent with the zero value in GR [10]. (The zero rest mass of photons is a Special Relativistic or GR concept but is not a Newtonian concept). Consequently, we have the equation of the light ray trajectory exactly as in GR:

$$
u^{\prime}+\frac{d^{2} u^{\prime}}{d \varphi^{2}}=3 m u^{\prime 2}
$$

Thus Eqs.(17) and (23), respectively, seem to provide the same GR results as far as the weak field tests for the perihelion advance and the bending of light are concerned. To examine the situation more closely, recall what steps were involved. The first step is the radial rescaling $u \rightarrow u^{\prime}$ which has no physical import. The second step is that, in arriving at Eq.(16), we have ignored terms like $O\left(m^{2} u^{\prime 2}\right)$ on numerical grounds. Note that it is only Eq.(15) per se, and not Eq.(17), that inverts exactly to the original Eq.(3) in the $(u, \varphi)$ coordinates describing the inverse square law. As we see, Eq.(17) produces an additional $3 m u^{\prime 2}$ term! Strictly speaking, Eq.(17) is approximate to the extent we ignored the smaller terms compared to unity (of the order of $10^{-16}$ and less!) in arriving at it. Treating this Eq.(17) as an exact equation means that we are retaining the cubic additional term as the only perturbation while disregarding the remaining smaller perturbations. This is the only nontrivial step we have adopted in the above computation.

If we had retained the smaller terms in Eq.(15), then it could tell the original situation: the exact Newtonian orbits. It is our nontrivial, but numerically justified, omission of the smaller terms that has brought forth equations similar to those in GR. Thus the exact solution of Eq.(15) is still a Keplerian ellipse but its expression does not look as familiar as in Eq.(4). Instead, in the primed coordinates, it looks like

$$
u^{\prime}=u \Phi(u)=\frac{\Phi(u)}{p}(1+e \cos \varphi) .
$$

where $u$ is given by Eq.(4). Expressions might differ in looks depending on the choice of coordinates, but the orbital shapes do not change.

One might think that though Eq.(17) looks different from Eq.(15), it still represents a Keplerian ellipse in the $\left(u^{\prime}, \varphi\right)$ coordinates. This is not the case since Eq.(17) is now nonlinear. We can find its solution by standard procedures starting with the zeroth order solution $u_{0}^{\prime}=\frac{1}{p}(1+e \cos \varphi)$ which is the solution of $u^{\prime}+\frac{d^{2} u^{\prime}}{d \varphi^{2}}=\frac{1}{p}$. Eq.(17) then gives the observed perihelion advance as $\frac{6 \pi M}{p}$. [Note that if one starts with the same $u_{0}^{\prime}$ in Eq.(15) or its second derivative 
form, one would eventually end up with Eq.(24) as the final solution]. Likewise, the exact equation for a straight line is

$$
u^{\prime}=\frac{1}{R} \Phi(u) \cos \varphi
$$

where $R$ is the distance from the origin. To zeroth order, $u_{0}^{\prime}=\frac{\cos \varphi}{R}$ is a solution of $u^{\prime}+\frac{d^{2} u^{\prime}}{d \varphi^{2}}=0$. By usual methods again with Eq.(23), one finds a total observed bending of light rays $\triangle \varphi \simeq \frac{4 M}{R}$.

The procedure leading to Eq.(17) has some similarity with that in GR. In the curved spacetime of GR, one needs to consider coordinate independent proper length $l$ instead of the radial coordinate $r$. Thus, in the Schwarzschild metric, $l$ is given by

$$
\begin{gathered}
l=\int \frac{d r}{\sqrt{1-\frac{2 m}{r}}}= \\
\frac{\sqrt{r}(-2 m+r)+2 m \sqrt{2 m-r} \arctan \sqrt{r /(2 m-r)}}{\sqrt{r\left(1-\frac{2 m}{r}\right)}}
\end{gathered}
$$

In terms of $(l, \varphi)$ coordinates, the GR Eq.(19) can not maintain its form or assume another exact closed form due to the fact that $r$ can not be expressed in terms of $l$ in a closed form. However, in the weak field region, $r \simeq l$, and we can maintain the form of Eq.(19) as it is, while ignoring higher order terms in $l$. In the present calculation, the background is Euclidean and so we can express $l$, using Eq.(8), as $l=\int d r=\int \Phi\left(r^{\prime}\right) \Omega^{-1}\left(r^{\prime}\right) d r^{\prime}$. In our calculation, we have ignored higher order terms in $u^{\prime}$ in the weak field region so that $r \simeq r^{\prime}$ and we ended up with Eq.(17).

Can we physically interpret our nontrivial step as a modification of the Newtonian force law? In this context, it is to be noted that, historically, Newton himself attempted to modify his force law to explain some phenomenon (for details, see Ref. [5]). One might also recall other efforts, for instance, Sommerfeld's calculation [11] for the precession of an electron in a Coulomb potential due to a proton $(Z=1)$ :

$$
\frac{d}{d t}\left(\frac{m_{0} \vec{v}}{\sqrt{1-v^{2}}}\right)=\frac{Z e^{2}}{r^{2}} \widehat{r}
$$

where $\widehat{r}$ is a unit vector in the radial direction and $e$ is the electronic charge. However, it produces only $(1 / 6)$ th of the observed perihelion advance of planets if the Coulomb potential on the right is replaced by the Newtonian potential. One could try the above special relativistic equation with another kind of force law on the right [12]

$$
\frac{d}{d t}\left(\frac{m_{0} \vec{v}}{\sqrt{1-v^{2}}}\right)=\frac{M m_{0}}{r^{2}\left(1-v^{2}\right)^{\frac{5}{2}}} \widehat{r}
$$

where $v^{2}=\dot{r}^{2}+r^{2} \dot{\varphi}^{2}$ does produce the observed perihelion advance, but the difficulty is that its first integral does not produce the conserved relativistic energy. This is understandable because the potential is velocity dependent. 
Coming back to our calculation, one might say that Eq.(17) [which is the same as Eq.(19)] corresponds to a potential $V(r)=-\frac{M}{r}-\frac{M}{r^{3}}$ but then the last term leads to a dimensional mismatch (see ref.[5]). Because of this, our procedure can not be interpreted as a modification of the Newtonian force law. Also, there was absolutely no use of the concept of geometric curvature in the calculation; it was completely Euclidean.

Thus, we conclude that the similarity between Eqs.(17) and (19) is only a fortuitous though amusing coincidence; it is just a mirage resulting from the choice of coordinates. There is absolutely no reason to prefer $\left(u^{\prime}, \varphi\right)$ coordinates over others and in this case, the formal coincidence will be lost. Nonetheless, the procedure illustrates something of pedagogical importance in the treatment of differential equations: One should be watchful with smaller terms! Their removal can nonlinearize a given linear equation [like going from Eq.(15) to (17)] and conversely, their restoration can linearize a known nonlinear equation [like returning from Eq.(17) to (15)].

It is a pleasure to thank Guzel Kutdusova and Arunava Bhadra for useful discussions.

\section{References}

[1] F. de Felice, "On the gravitational field acting as an optical medium", Gen. Rel. Grav. 2, 347-357 (1971).

[2] A recent useful reformulation of the historical optical-mechanical analogy has been conceived by: J. Evans and M. Rosenquist, " $F=m a$ " optics", Am. J. Phys. 54, 876-883 (1986). Newton's laws of motion are obtained directly from Fermat's principle in: M. Rosenquist and J. Evans, "The classical limit of quantum mechanics from Fermat's principle and the de Broglie relation", Am. J. Phys. 56, 881-882 (1988). For application to gradient-index lenses, see: J. Evans, "Simple forms for equations of rays in gradient-index lenses", Am. J. Phys. 58, 773-778 (1990). A short yet very illuminating discussion of the limitations and significance of the analogy may be found in: J. A. Arnaud, "Analogy between optical rays and non-relativistic particle trajectories: A comment", Am. J. Phys. 44, 1067-1069 (1976).

[3] The above reformulation has been applied in the GR context by: K.K. Nandi and A. Islam, "On the optical-mechanical analogy in general relativity", Am. J. Phys. 63, 251-256 (1995). The method has been extended to rotating bodies by: P.M. Alsing, "The optical-mechanical analogy for stationary metrics in general relativity", Am. J. Phys. 66, 779-790 (1998). The medium approach yields a possible observable effect in a new setting. This is discussed in: K. K. Nandi, Yuan-Zhong Zhang, P. M. Alsing, J. C. Evans, and A. Bhadra, "Analogue of the Fizeau effect in an effective optical medium", Phys. Rev. D 67, 025002 (1-11) (2003). Historically, Einstein himself conjectured the idea of an equivalent optical medium (This is reported in Ref.[1]). However, to our knowledge, Sir A.S. Eddington seems to be the first to have calculated the bending of light rays by assuming an approximate index $n(r) \simeq 1-\frac{2 M}{r}$. This can be found in: Space, Time and Gravitation (Cambridge University, Cambridge, 1920), reissued in the Cambridge Science Classic Series, 1987, p.109.

[4] For a further extension of the analogy that covers both massive and massless particles as well as applications to Cosmology, see: J. Evans, K.K. Nandi, and A. Islam, "The optical-mechanical analogy in general relativity: New methods for the paths of light and of the planets", Am. J. Phys. 64, 
1404-1415 (1996). For a semiclassical application, interested readers may have a look at: J. Evans, P.M. Alsing, S. Giorgetti, and K.K. Nandi, "Matter waves in a gravitational field: An index of refraction for massive particles in general relativity", Am. J. Phys. 69, 1103-1110 (2001).

[5] G. Maneff, "La gravitation et le principe de l'egalité de l'action et de la réaction", Comptes Rendus Acad. Sci. Paris 178, 2159-2161 (1924). Maneff assumed a variable test mass, viz., $m_{0}=m_{0}^{\prime} \exp \left(\frac{M}{r}\right)$ where $m_{0}^{\prime}$ is an invariant. This led to a force law: $F=\frac{M m_{0}}{r^{2}}\left(1+\frac{3 M}{r}\right)$. For a complete reference of the works by Maneff, see the interesting article by R.I. Ivanov and E.M. Prodanov [Arxiv: gr-qc/0504025. The authors also mention that Newton modified his potential law to $V(r)=A \frac{M}{r}+B \frac{M^{2}}{r^{2}}$ where $A$ and $B$ are constants, to explain the deviation of Moon's motion from the Keplerian laws. (Note the dimensional consistencies in both modifications.)

[6] F.R. Tangherlini, "Particle approach to the Fresnel coefficients", Phys. Rev. A 12, 139-147 (1975). See also: R. Tian and Z. Li, "The speed and apparent rest mass of photons in a gravitational field", Am. J. Phys. 58, 890-892 (1990).

[7] C. MØller, The Theory of Relativity, 2nd Ed. (Oxford University, Oxford, 1972), pp 498-501.

[8] W. Rindler, Essential Relativity, 2nd Ed. (Springer-Verlag, New York, 1977), p.143.

[9] S.K. Bose, An Introduction to General Relativity (Wiley Eastern, New Delhi, 1980), pp. 37-40.

[10] See, for instance, the treatise by S. Weinberg, Gravitation and Cosmology (John Wiley, New York, 1972), pp.186-187. If we start with the usual GR geodesic equations, then, in the low velocity, weak field limit, they reduce to $r^{2} \dot{\varphi} \simeq h_{0}$ and $\frac{1}{2}\left[\dot{r}^{2}+\frac{h_{0}^{2}}{r^{2}}\right]-\frac{M}{r} \simeq \frac{1-E}{2}$. For photons, $E=0$ so that for the total energy, we are left with a nonzero value $\frac{1}{2}$. If we start with the Newtonian equations, we instead get for light motion the value $\frac{1}{4}$ from Eq.(1) because $E_{0}=\frac{1}{2}$. The discrepant factor of 2 is actually a contribution from GR but it makes no difference to us as we have essentially started from the Newtonian theory. It is the nonzero value on the right of Eq.(1) for light that is consistent with the zero value in GR.

[11] Sommerfeld's calculation is discussed in: P.G. Bergmann, Introduction to the Theory of Relativity (Prentice-Hall, Englewood Cliffs, New Jersey, 1942).

[12] T.K. Ghoshal, K.K. Nandi, and S.K. Ghosal, "On the precession of the perihelion of Mercury", Indian J. Pure \& Appl. Math. 18, 194-199 (1987). 